TITLE:

\title{
Evaluation of extensional and torsional stiffness of single actin filaments by molecular dynamics analysis.
}

\section{AUTHOR(S):}

Matsushita, Shinji; Adachi, Taiji; Inoue, Yasuhiro; Hojo, Masaki; Sokabe, Masahiro

\section{CITATION:}

Matsushita, Shinji ... [et al]. Evaluation of extensional and torsional stiffness of single actin filaments by molecular dynamics analysis.. Journal of biomechanics 2010, 43(16): 31623167

ISSUE DATE:

2010-12-01

URL:

http://hdl.handle.net/2433/139249

\section{RIGHT:}

(C) 2010 Elsevier Ltd; This is not the published version. Please cite only the published version.; この論文は出版社版でありません。引用の際に は出版社版をご確認ご利用ください。 


\section{Evaluation of Extensional and Torsional Stiffness of \\ Single Actin Filaments by Molecular Dynamics Analysis}

Shinji Matsushita ${ }^{1,2}$, Taiji Adachi ${ }^{2,3}$, Yasuhiro Inoue ${ }^{1,2}$, Masaki Hojo ${ }^{1}$ and Masahiro Sokabe ${ }^{4,5}$

${ }^{1}$ Department of Mechanical Engineering and Science, Graduate School of Engineering, Kyoto University, Sakyo, Kyoto 606-8501, Japan

${ }^{2}$ Computational Cell Biomechanics Team, VCAD System Research Program, RIKEN, Hirosawa, Wako 351-0198, Japan

${ }^{3}$ Department of Biomechanics, Research Center for Nano Medical Engineering, Institute for Frontier Medical Sciences, Kyoto University, Sakyo, Kyoto 606-8507, Japan

${ }^{4}$ Department of Physiology, Graduate School of Medicine, Nagoya University, Syowa, Nagoya 466-8550, Japan

${ }^{5}$ ICORP/SORST, Cell Mechanosensing, Japan Science and Technology Agency, Honmachi, Kawaguchi 332-0012, Japan

\section{Corresponding Author:}

Taiji Adachi, Ph.D.

\section{Mailing Address:}

Taiji Adachi, Ph.D., Professor

Department of Biomechanics

Research Center for Nano Medical Engineering

Institute for Frontier Medical Sciences

Kyoto University

53 Kawahara-cho, Shogoin, Sakyo, Kyoto 606-8507, Japan

Tel \& Fax: +81 (75) 751-4853

E-mail: adachi@frontier.kyoto-u.ac.jp 
1 Abstract: It is essential to investigate the mechanical behaviour of cytoskeletal actin

filaments in order to understand their critical role as mechanical components in various cellular functional activities. These actin filaments consisting of monomeric molecules function in the thermal fluctuations. Hence, it is important to understand their mechanical behaviour on the microscopic scale by comparing the stiffness based on thermal fluctuations with the one experimentally measured on the macroscopic scale. In this study, we perform a large-scale molecular dynamics (MD) simulation for a half-turn structure of an actin filament. We analyse its longitudinal and twisting Brownian motions in equilibrium and evaluated its apparent extensional and torsional stiffness on the nanosecond scale. Upon increasing the sampling-window durations for analysis, the apparent stiffness gradually decreases and exhibits a trend to converge to a value that is close to the experimental value. This suggests that by extrapolating the data obtained in the MD analysis, we can estimate the experimentally determined stiffness on the microsecond to millisecond scales. For shorter temporal scales, the apparent stiffness is larger than experimental values, indicating that fast, local motions of the molecular structure are dominant. To quantify the local structural changes within the filament on the nanosecond scale and investigate the molecular mechanisms, such as the binding of the actin-regulatory proteins to the filaments, it is preferable to analyse the mechanical behaviour on the nanometre and nanosecond scales using MD simulation.

Keywords: Actin filament, Extensional and torsional stiffness, Thermal fluctuation, Molecular dynamics simulation, Computational biomechanics, Cell mechanics 


\section{Introduction}

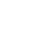

Several experimental studies have successfully estimated the mechanical properties of actin filaments (Kishino and Yanagida, 1988; Janmey et al., 1990), the most abundant component of cytoskeleton. They play critical mechanical roles in various cellular activities, such as migration, cell division and shape control (Svitkina et al., 1997; Watanabe and Mitchison, 2002; Pollard and Borisy, 2003; Pollard and Berro, 2009; Adachi et al., 2009).

\section{Using direct nanomanipulation techniques for single actin filaments, the 1- $\mu \mathrm{m}-\mathrm{long}$} extensional stiffness was determined to be $0.0437 \pm 0.0046$ [N/m] (Kojima et al., 1994). In addition, visualization of rotational beads attached to actin filaments (Tsuda et al., 1996) and measurements of phosphorescence anisotropy (Prochniewicz et al., 2005) made it possible to evaluate the torsional stiffness per unit length of the filament, which ranges from $2.3 \times 10^{-27}$ $\left[\mathrm{N} \cdot \mathrm{m}^{2}\right]$ (Prochniewicz et al., 2005) to $8.0 \times 10^{-26}\left[\mathrm{~N} \cdot \mathrm{m}^{2}\right]$ (Tsuda et al., 1996).

In these experiments, the length of the measured filaments was on the micrometre scale, and the temporal scales on which the dynamic motions were observed ranged from microsecond to millisecond. However, from a more microscopic viewpoint, the filaments have a double-helix structure on the nanometre scale with a period of $\sim 650 \AA$ (Holmes et al., 1990; Oda et al., 2009). In the physiological environment, they are exposed to thermal fluctuations yielding fast, local motions of the structure on temporal scales ranging from picosecond to nanosecond. In other words, vibrating motions of atoms in the molecule lead to local motions of the actin subunits, which then result in longitudinal and twisting motions of the filament. These microscopic dynamics of the molecular structure are the basis of 
1 determining the macroscopic mechanical properties of the filaments when they are observed

in the longer temporal scale; however, the temporal scale, which is long enough for capturing the mechanical properties comparable with the macroscopic properties obtained in the experiment, has not been investigated yet.

To analyse the mechanical behaviour of biomolecules at the molecular structural level, numerical simulations based on the molecular dynamics (MD) method have been extensively used and provided us with insights into several biologically relevant problems in various biomolecular systems (Wriggers and Schulten, 1997, 1999; Lu et al., 1998; Isralewitz et al., 2001; Vogel and Sheetz, 2006). For the biological filaments that build up biological materials, MD simulations were also applied to obtain information about their biological and physical properties (Buehler and Yung, 2009). For example, the mechanical properties of amyloid fibrils associated with Alzheimer's disease have been analyzed (Paparcone et al., 2010). For the cytoskeletal filaments, the bottom-up nanomechanical analysis has been performed from the viewpoint of multi-scale mechanics. For example, this analysis was performed for the human vimentin dimmer and tetramer, which constitute the intermediate filaments (Qin et al., 2009b, a).

For the actin monomers (Dalhaimer et al., 2008; Pfaendtner et al., 2009) and filaments (Chu and Voth, 2006, 2005; Pfaendtner et al., 2010), MD simulations have successfully analyzed their structural and dynamic properties. For example, the effect of the DB loop on the nucleotide-binding cleft has been investigated (Pfaendtner et al., 2010; Chu and Voth, 2006, 2005). Using the MD method, we can quantitatively observe the structural dynamics of 
1 the actin molecules and evaluate the stiffness of actin filaments based on their thermal

2 fluctuations.

In this study, we quantitatively evaluate the extensional and torsional stiffness of single actin filaments on the nanosecond scale by analyzing the thermal fluctuations of the molecular structure using the MD simulation, and compare the results with experimental data. We construct a model of the half-turn structure of an actin filament in an ionic solvent and analyse its longitudinal and twisting Brownian motions in equilibrium using MD simulation.

We then evaluate both the extensional and torsional stiffness of the actin filament from the thermal fluctuations based on the principle of equipartition of energy.

\section{Methods}

\section{Simulation procedures}

The 2.0- $\AA$ resolution X-ray crystallographic structure of actin filament was obtained from the Protein Data Bank (PDB code: 1MVW) (Holmes et al., 1990; Chen et al., 2002). From an original structure derived from rabbit skeletal muscle with six myosins, an actin filament structure consisting of 14 actin subunits was extracted as shown in Fig. 1. Then, ADP was placed into the individual actin subunits in the filament, in which the coordinates of the ADP were obtained from the ADP-bound monomer structure (PDB code: 1J6Z) (Otterbein et al., 2001). The actin filament structure of dimensions $\left(97 \times 98 \times 423 \AA^{3}\right)$ was placed into a water box of dimensions $\left(117 \times 118 \times 473 \AA^{3}\right)$, in which we utilized the TIP3P model for water molecules (Jorgensen et al., 1983). To the water box, $\mathrm{Na}^{+}$and $\mathrm{Cl}^{-}$counter ions were added to 
1 reach a concentration of $30 \mathrm{mM}$. The total system consisted of 496,434 atoms; 80,836 actin

2 filament atoms, 415,212 water atoms, $236 \mathrm{Na}^{+}$ions and $150 \mathrm{Cl}^{-}$ions.

MD simulation was performed using NAMD 2.6 (Kale et al., 1999) with the

CHARMM27 force field for proteins (MacKerell et al., 1998). We used periodic boundary conditions for simulations in which the van der Waals interactions were calculated with a cutoff of $13 \AA$ and the electrostatic interactions were calculated using the Particle Mesh Ewald method (Darden et al., 1993). A multiple time-stepping algorithm (Schlick et al., 1999) was used with a 2-fs step.

MD simulations were performed in an NPT ensemble (pressure $=1 \mathrm{~atm}$, temperature $=$ $310 \mathrm{~K})$, in which the pressure was controlled by the hybrid Nosé-Hoover Langevin piston method (Hoover, 1985; Martyna et al., 1992) and the temperature was controlled using Langevin dynamics. After relaxing the water molecules for 200 ps keeping all the protein atoms fixed, a free dynamics simulation for the entire system was performed for $12 \mathrm{~ns}$ to obtain the equilibrium structure.

\section{Evaluation of stiffness}

Length of the actin filament structure, $L(t)$, is defined as

$$
L(t)=z_{\mathrm{plus}}(t)-z_{\text {minus }}(t)
$$

where $z_{\text {plus }}(t)$ is the position in the $z$-axis of the centre of mass of G-actins, $\mathrm{G}_{13}$ and $\mathrm{G}_{14}$, at the plus-end, and $z_{\text {minus }}(t)$ is that of the G-actins, $\mathrm{G}_{1}$ and $\mathrm{G}_{2}$, at the minus-end, as shown in Fig. 2 (left).

Twist angle of the actin filament structure, $\Theta(t)$, is defined as 


$$
\Theta(t)=\cos ^{-1}\left(\frac{n_{\mathrm{plus}}(t)}{\left|n_{\mathrm{plus}}(t)\right|} \bullet \frac{n_{\text {minus }}(t)}{\left|n_{\text {minus }}(t)\right|}\right),
$$

2 where

3

4

Using the law of equipartition of energy, the extensional spring constant $k_{\mathrm{ext}}^{\Delta t}(t)$ and torsional spring constant $k_{\text {tor }}^{\Delta t}(t)$ of the filament can be estimated from the variances of the filament length $L(t)$ and twist angle $\Theta(t)$ during the sampling-window duration $\Delta t$, as

$$
\begin{aligned}
& \frac{1}{2} k_{\mathrm{ext}}^{\Delta t}(t)<\left(L(t)-<L(t)>_{\Delta t}\right)^{2}>_{\Delta t}=\frac{1}{2} k_{\mathrm{B}} T, \\
& \frac{1}{2} k_{\mathrm{tor}}^{\Delta t}(t)<\left(\Theta(t)-<\Theta(t)>_{\Delta t}\right)^{2}>_{\Delta t}=\frac{1}{2} k_{\mathrm{B}} T,
\end{aligned}
$$

and $\boldsymbol{P}_{\mathrm{Gi}}(t)$ is the position vector of the centre of mass of G-actin $\mathrm{G}_{\mathrm{i}}$ projected onto the $x-y$ frame.

where $k_{\mathrm{B}}$ is the Boltzmann constant, $T$ is the absolute temperature and \langle\rangle$_{\Delta t}$ indicates the average over a time $\left(t-\frac{\Delta t}{2} \leq t<t+\frac{\Delta t}{2}\right)$. In this study, the spring constants $k_{\mathrm{ext}}^{\Delta t}(t)$ and MD simulations. experimental studies (Kojima et al., 1994; Prochniewicz et al., 2005). Extensional stiffness plane, as shown in Fig. 2 (right). For the analysis, the filament length $L(t)$ and twist angle $\Theta(t)$ are determined by aligning the longitudinal axis of the filament along the $z$-direction in each $k_{\text {tor }}^{\Delta t}(t)$ of the filament for $\Delta t=0.5,1.0,2.0,4.0,8.0$ and $16.0 \mathrm{~ns}$ were evaluated based on the

In this study, we evaluate the stiffness by using the conventional units, as used in previous 
1 has been calculated as the value per $1-\mu \mathrm{m}$ length, and torsional stiffness as the value per unit

2 length. From the spring constants $k_{\mathrm{ext}}^{\Delta t}(t)$ and $k_{\mathrm{tor}}^{\Delta t}(t)$, the $1-\mu \mathrm{m}$-long apparent extensional

$3 \quad$ stiffness, $K_{\mathrm{ext}}^{\Delta t}(t)$, is given by

4

$$
K_{\mathrm{ext}}^{\Delta t}(t)=\frac{<L(t)>_{\Delta t}}{1 \mu \mathrm{m}} k_{\mathrm{ext}}^{\Delta t}(t)
$$

5

and the apparent torsional stiffness, $K_{\text {tor }}^{\Delta t}(t)$, per unit length of filament is given by

$$
K_{\mathrm{tor}}^{\Delta t}(t)=<L(t)>_{\Delta t} k_{\mathrm{tor}}^{\Delta t}(t)
$$

\section{Results}

\section{Changes in filament length and twist angle} fluctuations of the actin filament, which are shown in Fig. 3. In this figure, the changes in the filament length $\langle L(t)\rangle_{25}$ ns over the 25 -ns period is $326.6 \AA$, and its standard deviation, $\sqrt{\left\langle\Delta L^{2}(t)\right\rangle_{12 \mathrm{~ns}}}=\sqrt{\left\langle\left(L(t)-\langle L(t)\rangle_{12 \mathrm{~ns}}\right)^{2}\right\rangle_{12 \mathrm{~ns}}}$, is $0.6 \AA$. Using these values, we estimate the average value of the twist angle $\langle\Theta(t)\rangle_{25}$ ns over a 25 -ns period is $182.2 \mathrm{deg}$, and its actin filament and determine its mechanical behaviour.

For the 25-ns equilibrium simulation, we monitored the longitudinal and twisting thermal filament length $L(t)$ and twist angle $\Theta(t)$ are plotted at intervals of $1 \mathrm{ps}$. The average value of the average extensional strain of the filament driven by thermal energy to be $\sim 0.2 \%$. Similarly, standard deviation, $\sqrt{\left\langle\Delta \Theta^{2}(t)\right\rangle_{25 \mathrm{~ns}}}=\sqrt{\left\langle\left(\Theta(t)-<\Theta(t)>_{25 \mathrm{~ns}}\right)^{2}>_{25 \mathrm{~ns}}\right.}$, is $6.6 \mathrm{deg}$. Thus, we estimate the twist angle per unit length of the filament driven by thermal energy to be $\sim 0.02$ $\operatorname{deg} / \AA$. These structural fluctuations driven by thermal energy characterize the stiffness of the 
1

2

3

4

5

\section{Changes in extensional and torsional stiffness}

Based on Eqs. (5) - (8), we estimated the extensional stiffness $K_{\mathrm{ext}}^{\Delta t}(t)$ and the torsional stiffness $K_{\text {tor }}^{\Delta t}(t)$ of the actin filament from the variances of the filament length and the twist angle. Figures $4 \mathrm{~A}$ and $4 \mathrm{~B}$ show the change over time in both the variance of the filament length $\left\langle\Delta L^{2}(t)\right\rangle_{\Delta t}$ and the extensional stiffness $K_{\mathrm{ext}}^{\Delta t}(t)$, plotted at intervals of $1 \mathrm{ps}$, determined for each sampling-window duration $\Delta t(=0.5,1.0,2.0,4.0,8.0$ and $16.0 \mathrm{~ns})$. For the longer sampling-window durations $\Delta t$, the extensional stiffness tends to decrease with increasing sampling-window duration $\Delta t$. Thus, on the shorter time scales, the filaments appear to exhibit much larger stiffness than that measured experimentally. To show the dependence of the apparent stiffness on the sampling-window duration $\Delta t$, the distribution of the extensional stiffness $K_{\text {ext }}^{\Delta t}(t)$ is plotted against the sampling-window duration $\Delta t$, as shown in Fig. 4C.

Similarly, the dynamics of the variances of the twist angle $\left\langle\Delta \Theta^{2}(t)\right\rangle_{\Delta t}$, the torsional stiffness $K_{\text {tor }}^{\Delta t}(t)$ and the distribution of torsional stiffness exhibit a tendency similar to that found for the extensional stiffness, as shown in Fig. 5.

As plotted by solid circles in Figs. 4C and 5C, the average values of both extensional and torsional stiffness gradually decrease with increasing sampling-window durations $\Delta t$ and exhibit a trend to converge close to values that are comparable to the experimental data (Kojima et al., 1994; Prochniewicz et al., 2005) indicated by dashed lines. The increase in apparent stiffness for shorter sampling-window durations is mainly due to a limitation in capturing the structural fluctuations of the entire filament. In other words, the fluctuations of the entire filament can be observed through the longer sampling-window durations, whereas 
1 only instantaneous fluctuations of local parts such as the actin subunits and subdomains can

2 be observed through the shorter sampling-window durations, resulting in the larger apparent

3 stiffness. The extensional stiffness estimated for the longest sampling-window duration $\Delta t=$

$416.0[\mathrm{~ns}]$ in the MD simulation, $K_{\text {ext }}^{\Delta t=16.0 \mathrm{~ns}}=0.035 \pm 0.004[\mathrm{~N} / \mathrm{m}]$, is in good agreement with

5 the experimentally measured values, $K_{\text {ext }}^{\exp }=0.0437 \pm 0.0046[\mathrm{~N} / \mathrm{m}]$ (Kojima et al., 1994).

6 The torsional stiffness for $\Delta t=16.0[\mathrm{~ns}], \quad K_{\mathrm{tor}}^{\Delta t=16.0 \mathrm{~ns}}=(1.1 \pm 0.1) \times 10^{-26}\left[\mathrm{~N} \cdot \mathrm{m}^{2} / \mathrm{radian}\right]$, is

7 comparable but slightly larger than the experimental value, $K_{\text {tor }}^{\exp }=(0.23 \pm 0.10) \times 10^{-26}$

$8 \quad\left[\mathrm{~N} \cdot \mathrm{m}^{2} /\right.$ radian $]$ (Prochniewicz et al., 2005), which was determined on the microsecond scale.

9 Consequently, these results suggest that by extrapolating the data obtained in the MD analysis

to longer time scale, we can adequately estimate the filament stiffness observable in the experiments.

To investigate the effects of free ends of the filament, we extracted trajectory data from the central part of the filament consisting of 10 actin subunits without the end monomers $\mathrm{G}_{1}$, $\mathrm{G}_{2}, \mathrm{G}_{13}$ and $\mathrm{G}_{14}$, and evaluated the stiffness of the central part. As a result, although the extensional and torsional stiffness of the central part exhibited slightly larger values than those of the entire filament containing free ends, both stiffnesses are comparable to the experimental values, as shown in Figs. 4C and 5C.

\section{Discussion}

In this study, we evaluated the extensional and torsional stiffnesses of the actin filament on the basis of thermal fluctuations, in nanosecond scales. The results demonstrate that with 
1 increasing analyzed sampling-window duration, the apparent stiffness evaluated on the

2

nanosecond scale gradually decreases and exhibits a trend to converge to a value that is close to the experimental value. That is, we determined the length of a temporal scale sufficient for capturing the mechanical properties comparable with the macroscopic properties evaluated in the experiments.

The estimated extensional stiffness of the half-turn filament of $\sim 326.6 \AA$ for the longest sampling-window duration $\Delta t=16.0$ [ns] is in good agreement with the experimentally measured stiffness of the micrometre-long filaments. This result indicates that the half-turn filament could be regarded as a basic unit whose extensional stiffness adequately represents the stiffness of long filaments. The torsional stiffness of the half-turn filament is comparable but slightly larger than the stiffness of the micrometre-long filaments. This indicates that the sampling-window duration $\Delta t=16.0[\mathrm{~ns}]$ is slightly shorter than that required for evaluating the torsional stiffness of long filaments, in which it is necessary to extrapolate the data to a longer time scale. These results may lead to state that the mechanical properties on the macroscopic scale could be adequately captured over the different spatiotemporal scales by examining the mechanical behaviour of the half-turn filament structure, indicating the validity of thermal fluctuation analyses of the actin filament using MD simulations.

For shorter sampling-window durations $(\Delta t<8.0[\mathrm{~ns}])$, the apparent extensional and torsional stiffness of the filament are larger than the experimentally measured values, indicating that, in the shorter durations, only a part of the filament fluctuations is observed. That is, the shorter sampling-window duration cannot capture the mechanical properties of the 
1 entire filament adequately. Despite this, the result obtained in the shorter duration will be

essential, for example, when we consider the binding process of actin-regulatory proteins to

filaments, because the structural changes in the filament during the binding process are local events ranging from the amino-acid to subunit scales. These local structural changes in the binding process are measured only on adequately short temporal scales. Therefore, to investigate the binding process of actin-regulatory proteins to filament, it is important to observe the local structural changes of actin filaments on the short temporal scales.

Some proteins binding to the filaments (Isenberg et al., 1980; Pollard and Cooper, 1986) are known as important regulators that influence the global reorganization of the actin cytoskeleton (Naruse and Sokabe, 1993; Sato et al., 2000; Neidlinger-Wilke et al., 2001; Sato et al., 2005; Yamamoto et al., 2006) controlled by interactions among mechanical and biochemical factors (Theriot and Mitchison, 1991; Arber et al., 1998; Hayakawa et al., 2008; Okeyo et al., 2009). In the dynamic cytoskeletal reorganization process, the binding of actin-regulatory proteins to the filaments causes molecular-level structural changes in the double-helix structure. One of the important actin-regulatory proteins present in all eukaryotic cells is cofilin, which is known as the actin depolymerizing factor. It binds along the length of the filament, twisting a little more tightly (McGough et al., 1997), and increasing the torsional flexibility (Prochniewicz et al., 2005). Therefore, the MD analysis allows us to measure the local structural changes and elucidates the underlying molecular mechanisms of the binding process.

We evaluated the stiffness by using the actin filament model obtained by Holmes et al. 
1 (1990). Recently, Oda et al. (2009) have obtained the refined filamentous actin structure. The

2 Oda filament model has been constructed with a similar helical arrangement as the Holmes

3 model but with a different actin subunit structure. MD simulations of the actin filaments

4 conducted by Pfaendtner et al. (2010) on the basis of the structure models by Holmes (1990)

5 and Oda (2009) revealed that two different initial structures did not converge to the same

6 structure within the MD simulation time. This result implies that the initial structure is critical

7 for the biomolecular functions such as molecular interactions in the nucleotide binding cleft

8 (Pfaendtner et al., 2010). Contrary to this situation, macroscopic mechanical functions, such

9 as the persistence length of the filament, agrees well with the experimental values without a significant difference between the Oda and Holmes filament models (Pfaendtner et al., 2010).

This result suggests that the structural difference between the two models may not result in a significant difference in the macroscopic mechanical properties of the filaments such as extensional and torsional stiffness estimated in this study on the basis of the MD simulation. Importantly, Pfaendtner et al. (2010) revealed that the conformation of the DB loop is a more significant determinant of the persistence length. Therefore, the effects of the DB loop on the filament extensional and torsional stiffness need to be analyzed in detail for the next step. Assuming the filament to be a homogeneous rod with a circular cross section, the Young's modulus is determined as $E=k_{\text {ext }} L / A$, where $A$ is an equivalent cross-sectional area of the 
1 contour of the filament cross section based on the filament atomic model (Holmes et al.,

2 1990), if the area in the contour is filled homogeneously (Kojima et al., 1994). In this study,

3 Young's modulus $E$ is estimated as $1.4 \times 10^{9} \mathrm{~N} / \mathrm{m}^{2}$, which is on the same order as other

4 proteins such as vimentin $\left(5.4 \times 10^{8} \mathrm{~N} / \mathrm{m}^{2}\right)$ (Qin et al., $\left.2009 \mathrm{~b}\right)$, tubulin $\left(2.2 \times 10^{9} \mathrm{~N} / \mathrm{m}^{2}\right)($ Sept

5 and MacKintosh, 2010) and tropocollagen $\left(7.0 \times 10^{9} \mathrm{~N} / \mathrm{m}^{2}\right)$ (Uzel and Buehler, 2009).

In experiments to measure the stiffness, the actin filaments are usually slightly tensioned

$7 \quad$ (Kishino and Yanagida, 1988) and labelled with fluorescence markers conjugate with

8 phalloidin. Phalloidin is known as a structural stabilizer for actin filaments; therefore, its

9 binding may result in an increase in the filament stiffness (Chu and Voth, 2006). In contrast,

MD analyses evaluate the stiffness under ideal conditions without artifacts such as tension and

the binding of markers. Therefore, we expect that the method proposed in this study of

estimating the filament stiffness with MD analysis will be applied over a wide range to

evaluate other filamentous proteins. Furthermore, the steered MD simulations, which enable

us to control positions and velocities of some specified atoms by applying external steering

such as $\alpha$-actinin rod (Golji et al., 2009), intermediate filaments (Qin et al., 2009b) and quantitatively observe the structural dynamics of the actin molecules. 
1 (Ming et al., 2003; Yamaoka and Adachi, 2007, 2010). Based on CG simulations, we can

2 analyse the behaviour of actin filaments on larger spatiotemporal scales in comparison to the MD analysis. For example, normal mode analysis (Tirion and Benavraham, 1993) was applied to investigate the structural properties of the filaments combined with the substructure synthesis method (Ming et al., 2003) applied for the actin filament. In CG and continuum simulations, the stiffness in particular, is a fundamental parameter in filament dynamics, because the mechanical behaviour of actin filaments and their interactions with actin regulatory proteins strongly depend on the mechanical properties of the filament, such as extensional and torsional stiffness. Therefore, MD analysis, by which the dynamics can be evaluated over a wide range of time scales, gives us critical information for quantitative CG, continuum modelling and simulation for multiscale analysis of actin filament dynamics. The analysis for larger-scale structures in a longer temporal scale will provide challenging opportunities for better understanding of the mechanical behaviour of the actin filaments as they interact with the other filaments and binding proteins that produce network dynamics with higher-order hierarchical structures. Therefore, large-scale simulations will provide further insight into more complicated phenomena that cannot be easily approached by the current experiments.

\section{Conflict of Interest}

None. 


\section{Acknowledgement}

2

This research was partly supported by Research and Development of the Next-Generation

3 Integrated Simulation of Living Matter, a part of the Development and Use of the

4 Next-Generation Supercomputer Project, and by Grant-in-aid for Specially Promoted

5 Research (20001007) from the Ministry of Education, Culture, Sports, Science and

6 Technology (MEXT), and the Japan Society for the Promotion of Science (JSPS) Fellows for

\section{$7 \quad$ Young Scientists.}

8 


\section{References}

Adachi, T., Okeyo, K.O., Shitagawa, Y., Hojo, M., 2009, Strain field in actin filament network in lamellipodia of migrating cells: Implication for network reorganization. Journal of Biomechanics 42, 297-302.

Arber, S., Barbayannis, F.A., Hanser, H., Schneider, C., Stanyon, C.A., Bernard, O., Caroni, P., 1998, Regulation of actin dynamics through phosphorylation of cofilin by LIM-kinase. Nature 393, 805-809.

Buehler, M.J., Yung, Y.C., 2009, Deformation and failure of protein materials in physiologically extreme conditions and disease. Nature Materials 8, 175-188.

Chen, L.F., Winkler, H., Reedy, M.K., Reedy, M.C., Taylor, K.A., 2002, Molecular modeling of averaged rigor crossbridges from tomograms of insect flight muscle. Journal of Structural Biology 138, 92-104.

Chu, J.W., Voth, G.A., 2005, Allostery of actin filaments: Molecular dynamics simulations and coarse-grained analysis. Proceedings of the National Academy of Sciences of the United States of America 102, 13111-13116.

Chu, J.W., Voth, G.A., 2006, Coarse-grained modeling of the actin filament derived from atomistic-scale simulations. Biophysical Journal 90, 1572-1582.

Dalhaimer, P., Pollard, T.D., Nolen, B.J., 2008, Nucleotide-mediated conformational changes of monomeric actin and Arp3 studied by molecular dynamics simulations. Journal of Molecular Biology 376, 166-183.

Darden, T., York, D., Pedersen, L., 1993, Paticle mesh ewald - an N.log(N) method for ewald sums in large systems. Journal of Chemical Physics 98, 10089-10092.

Gautieri, A., Vesentini, S., Montevecchi, F.M., Redaelli, A., 2008, Mechanical properties of physiological and pathological models of collagen peptides investigated via steered molecular dynamics simulations. Journal of Biomechanics 41, 3073-3077.

Golji, J., Collins, R., Mofrad, M.R.K., 2009, Molecular Mechanics of the alpha-Actinin Rod Domain: Bending, Torsional, and Extensional Behavior. Plos Computational Biology $5,-$.

Hayakawa, K., Tatsumi, H., Sokabe, M., 2008, Actin stress fibers transmit and focus force to activate mechanosensitive channels. Journal of Cell Science 121, 496-503.

Holmes, K.C., Popp, D., Gebhard, W., Kabsch, W., 1990, Atomic model of the actin filament. Nature 347, 44-49.

Hoover, W.G., 1985, Canonical dynamics - equilibrium phase-space distributions. Physical Review A 31, 1695-1697.

Isenberg, G., Aebi, U., Pollard, T.D., 1980, An actin-binding protein from acanthamoeba regulates actin filament polymerization and interactions. Nature 288, 455-459.

Isralewitz, B., Gao, M., Schulten, K., 2001, Steered molecular dynamics and mechanical functions of proteins. Current Opinion in Structural Biology 11, 224-230.

Janmey, P.A., Hvidt, S., Oster, G.F., Lamb, J., Stossel, T.P., Hartwig, J.H., 1990, Effect of ATP on actin filament stiffness. Nature 347, 95-99.

Jorgensen, W.L., Chandrasekhar, J., Madura, J.D., Impey, R.W., Klein, M.L., 1983, 
Comparison of simple potemtial functions for simulating liquid water. Journal of Chemical Physics 79, 926-935.

Kale, L., Skeel, R., Bhandarkar, M., Brunner, R., Gursoy, A., Krawetz, N., Phillips, J., Shinozaki, A., Varadarajan, K., Schulten, K., 1999, NAMD2: Greater scalability for parallel molecular dynamics. Journal of Computational Physics 151, 283-312.

Kishino, A., Yanagida, T., 1988, Force measurements by micromanipulation of a single actin filament by glass needles. Nature 334, 74-76.

Kojima, H., Ishijima, A., Yanagida, T., 1994, Direct measurement of stiffness of single actin-filaments with and without tropomyosin by in-vitro nanomanipulation. Proceedings of the National Academy of Sciences of the United States of America 91, 12962-12966.

Lu, H., Isralewitz, B., Krammer, A., Vogel, V., Schulten, K., 1998, Unfolding of titin immunoglobulin domains by steered molecular dynamics simulation. Biophysical Journal 75, 662-671.

MacKerell, A.D., Bashford, D., Bellott, M., Dunbrack, R.L., Evanseck, J.D., Field, M.J., Fischer, S., Gao, J., Guo, H., Ha, S., Joseph-McCarthy, D., Kuchnir, L., Kuczera, K., Lau, F.T.K., Mattos, C., Michnick, S., Ngo, T., Nguyen, D.T., Prodhom, B., Reiher, W.E., Roux, B., Schlenkrich, M., Smith, J.C., Stote, R., Straub, J., Watanabe, M., Wiorkiewicz-Kuczera, J., Yin, D., Karplus, M., 1998, All-atom empirical potential for molecular modeling and dynamics studies of proteins. Journal of Physical Chemistry B 102, 3586-3616.

Martyna, G.J., Klein, M.L., Tuckerman, M., 1992, Nose-Hoover chains - the canonical ensemble via continuous dynamics. Journal of Chemical Physics 97, 2635-2643.

McGough, A., Pope, B., Chiu, W., Weeds, A., 1997, Cofilin changes the twist of F-actin: Implications for actin filament dynamics and cellular function. Journal of Cell Biology 138, 771-781.

Ming, D.M., Kong, Y.F., Wu, Y.H., Ma, J.P., 2003, Simulation of F-actin filaments of several microns. Biophysical Journal 85, 27-35.

Naruse, K., Sokabe, M., 1993, Involvement of stretch-activated ion channels in $\mathrm{Ca}^{2+}$ mobilization to mechanical stretch endothelial-cells. American Journal of Physiology 264, C1037-C1044.

Neidlinger-Wilke, C., Grood, E.S., Wang, J.H.C., Brand, R.A., Claes, L., 2001, Cell alignment is induced by cyclic changes in cell length: studies of cells grown in cyclically stretched substrates. Journal of Orthopaedic Research 19, 286-293.

Oda, T., Iwasa, M., Aihara, T., Maeda, Y., Narita, A., 2009, The nature of the globular-to fibrous-actin transition. Nature 457, 441-445.

Okeyo, K.O., Adachi, T., Sunaga, J., Hojo, M., 2009, Actomyosin contractility spatiotemporally regulates actin network dynamics in migrating cells. Journal of Biomechanics 42, 2540-2548.

Otterbein, L.R., Graceffa, P., Dominguez, R., 2001, The crystal structure of uncomplexed actin in the ADP state. Science 293, 708-711.

Paparcone, R., Keten, S., Buehler, M.J., 2010, Atomistic simulation of nanomechanical 
properties of Alzheimer's A beta(1-40) amyloid fibrils under compressive and tensile loading. Journal of Biomechanics 43, 1196-1201.

Pfaendtner, J., Branduardi, D., Parrinello, M., Pollard, T.D., Voth, G.A., 2009, Nucleotide-dependent conformational states of actin. Proceedings of the National Academy of Sciences of the United States of America 106, 12723-12728.

Pfaendtner, J., Lyman, E., Pollard, T.D., Voth, G.A., 2010, Structure and Dynamics of the Actin Filament. Journal of Molecular Biology 396, 252-263.

Pollard, T.D., Berro, J., 2009, Mathematical models and simulations of cellular processes based on actin filaments. Journal of Biological Chemistry 284, 5433-5437.

Pollard, T.D., Borisy, G.G., 2003, Cellular motility driven by assembly and disassembly of actin filaments. Cell 112, 453-465.

Pollard, T.D., Cooper, J.A., 1986, Actin and actin-binding proteins - a critical-evaluation of mechanism and functions. Annual Review of Biochemistry 55, 987-1035.

Prochniewicz, E., Janson, N., Thomas, D.D., De La Cruz, E.M., 2005, Cofilin increases the torsional flexibility and dynamics of actin filaments. Journal of Molecular Biology 353, 990-1000.

Qin, Z., Kreplak, L., Buehler, M.J., 2009a, Hierarchical Structure Controls Nanomechanical Properties of Vimentin Intermediate Filaments. Plos One 4, e7294.

Qin, Z., Kreplak, L., Buehler, M.J., 2009b, Nanomechanical properties of vimentin intermediate filament dimers. Nanotechnology 20, 425101.

Sato, K., Adachi, T., Matsuo, M., Tomita, Y., 2005, Quantitative evaluation of threshold fiber strain that induces reorganization of cytoskeletal actin fiber structure in osteoblastic cells. Journal of Biomechanics 38, 1895-1901.

Sato, M., Nagayama, K., Kataoka, N., Sasaki, M., Hane, K., 2000, Local mechanical properties measured by atomic force microscopy for cultured bovine endothelial cells exposed to shear stress. Journal of Biomechanics 33, 127-135.

Schlick, T., Skeel, R.D., Brunger, A.T., Kale, L.V., Board, J.A., Hermans, J., Schulten, K., 1999, Algorithmic challenges in computational molecular biophysics. Journal of Computational Physics 151, 9-48.

Sept, D., Elcock, A.H., McCammon, J.A., 1999, Computer simulations of actin polymerization can explain the barbed-pointed end asymmetry. Journal of Molecular Biology 294, 1181-1189.

Sept, D., MacKintosh, F.C., 2010, Microtubule Elasticity: Connecting All-Atom Simulations with Continuum Mechanics. Physical Review Letters 104, - .

Shimada, Y., Adachi, T., Inoue, Y., Hojo, M., 2009, Coarse-grained modeling and simulation of actin filament behavior based on Brownian dynamics method. Molecular and Cellular Biomechanics 6, 161-173.

Svitkina, T.M., Verkhovsky, A.B., McQuade, K.M., Borisy, G.G., 1997, Analysis of the actin-myosin II system in fish epidermal keratocytes: Mechanism of cell body translocation. Journal of Cell Biology 139, 397-415.

Theriot, J.A., Mitchison, T.J., 1991, Actin microfilament dynamics in locomoting cells. Nature 352, 126-131. 
Tirion, M.M., Benavraham, D., 1993, Normal Mode Analysis of G-Actin. Journal of Molecular Biology 230, 186-195.

Tsuda, Y., Yasutake, H., Ishijima, A., Yanagida, T., 1996, Torsional rigidity of single actin filaments and actin-actin bond breaking force under torsion measured directly by in vitro micromanipulation. Proceedings of the National Academy of Sciences of the United States of America 93, 12937-12942.

Uzel, S.G.M., Buehler, M.J., 2009, Nanomechanical sequencing of collagen: tropocollagen features heterogeneous elastic properties at the nanoscale. Integrative Biology 1, 452-459.

Vogel, V., Sheetz, M., 2006, Local force and geometry sensing regulate cell functions. Nature Reviews Molecular Cell Biology 7, 265-275.

Watanabe, N., Mitchison, T.J., 2002, Single-molecule speckle analysis of Aactin filament turnover in lamellipodia. Science 295, 1083-1086.

Wriggers, W., Schulten, K., 1997, Stability and dynamics of G-actin: Back-door water diffusion and behavior of a subdomain 3/4 loop. Biophysical Journal 73, 624-639.

Wriggers, W., Schulten, K., 1999, Investigating a back door mechanism of actin phosphate release by steered molecular dynamics. Proteins-Structure Function and Genetics 35, 262-273.

Yamamoto, K., Sokabe, T., Matsumoto, T., Yoshimura, K., Shibata, M., Ohura, N., Fukuda, T., Sato, T., Sekine, K., Kato, S., Isshiki, M., Fujita, T., Kobayashi, M., Kawamura, K., Masuda, H., Kamiya, A., Ando, J., 2006, Impaired flow-dependent control of vascular tone and remodeling in P2X4-deficient mice. Nature Medicine 12, 133-137.

Yamaoka, H., Adachi, T., 2007, A study on mechanical behaviour of single actin filament through continuum modeling. Journal of Biomechanical Science and Engineering 2, S18.

Yamaoka, H., Adachi, T., 2010, Coupling between axial stretch and bending/twisting deformation of actin filaments caused by a mismatched centroid from the center axis. International Journal of Mechanical Science 52, 329-333. 


\section{Figure Legends}

(1)

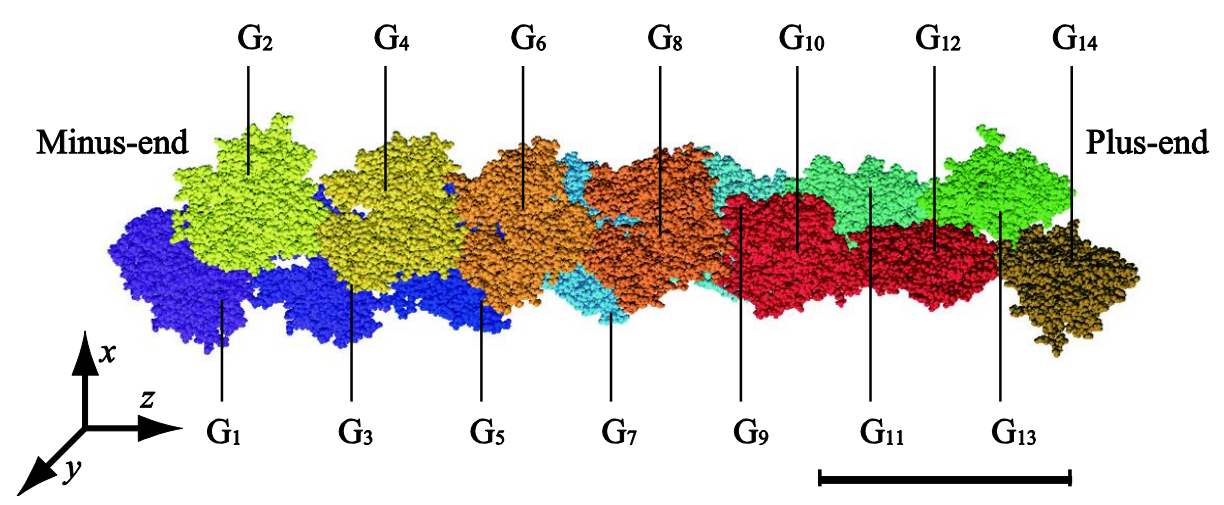

Figure 1: Double-helix structure of an actin filament consisting of 14 actin subunits. The total protein size is 80,836 atoms. The Cartesian coordinate axis $z$ is along the filament axis, and the orthogonal axes are $x$ and $y$. Each actin subunit is numbered from the minus-end as $\mathrm{G}_{1}$, $\mathrm{G}_{2}, \ldots, \mathrm{G}_{14}$. The size of the scale bar is $100 \AA$.

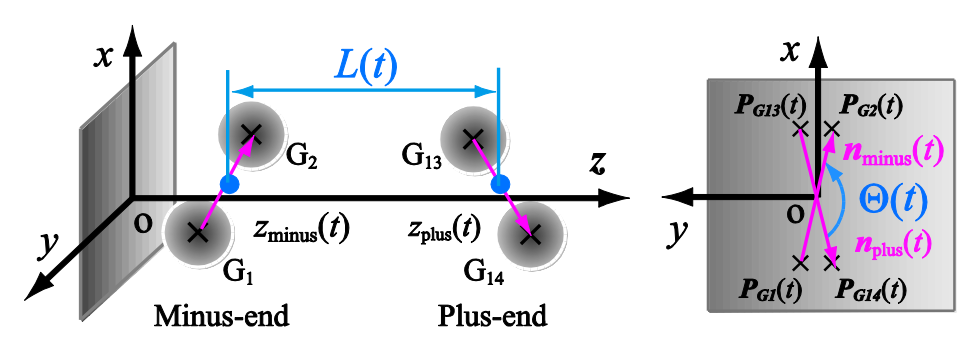

Figure 2: Definition of filament length and twist angle. The filament length $L(t)$ is defined by Eq. (1) as the distance between the plus- and minus-ends of the filament (left). The twist angle $\Theta(t)$ is defined by Eq. (2) as the angle between the vectors of the plus- and minus-ends (right). 

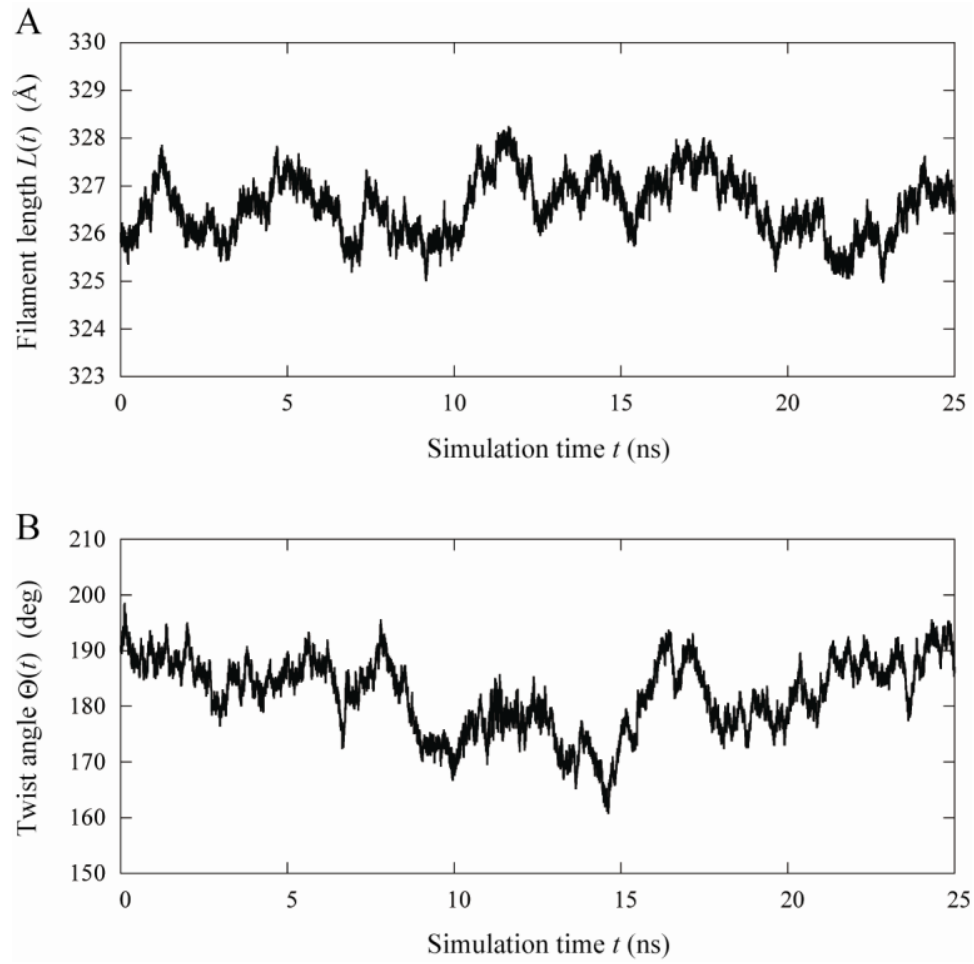

2 Figure 3: Structural changes during a 25-ns equilibrium subsequent to equilibrating

3 simulation for $12 \mathrm{~ns}$. (A) shows the change in filament length $L(t)$. The average filament

4 length is $326.6 \AA$. (B) shows the change in twist angle $\Theta(t)$. The average twist angle is 182.2 5 deg. 

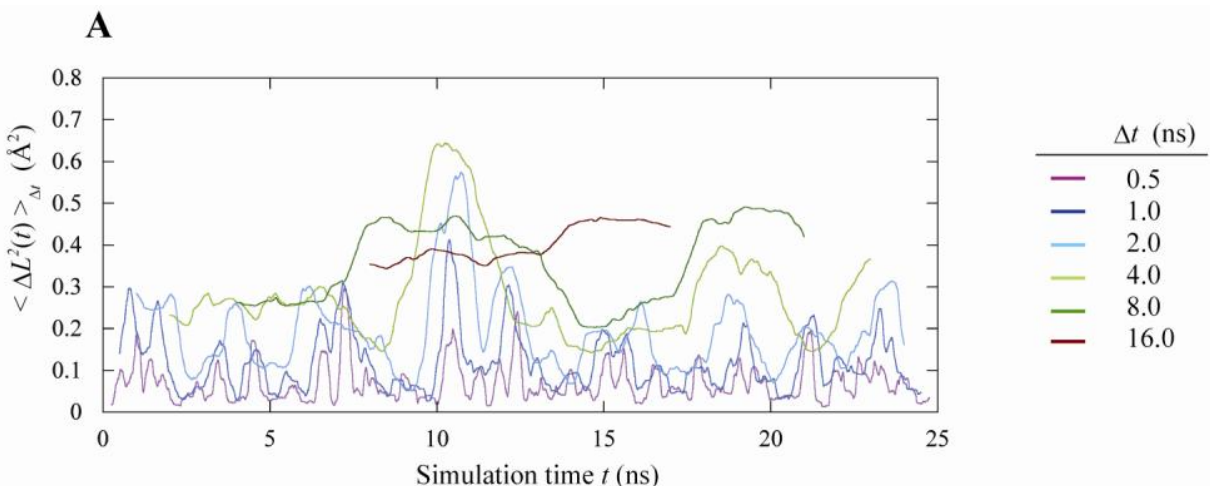

B

C
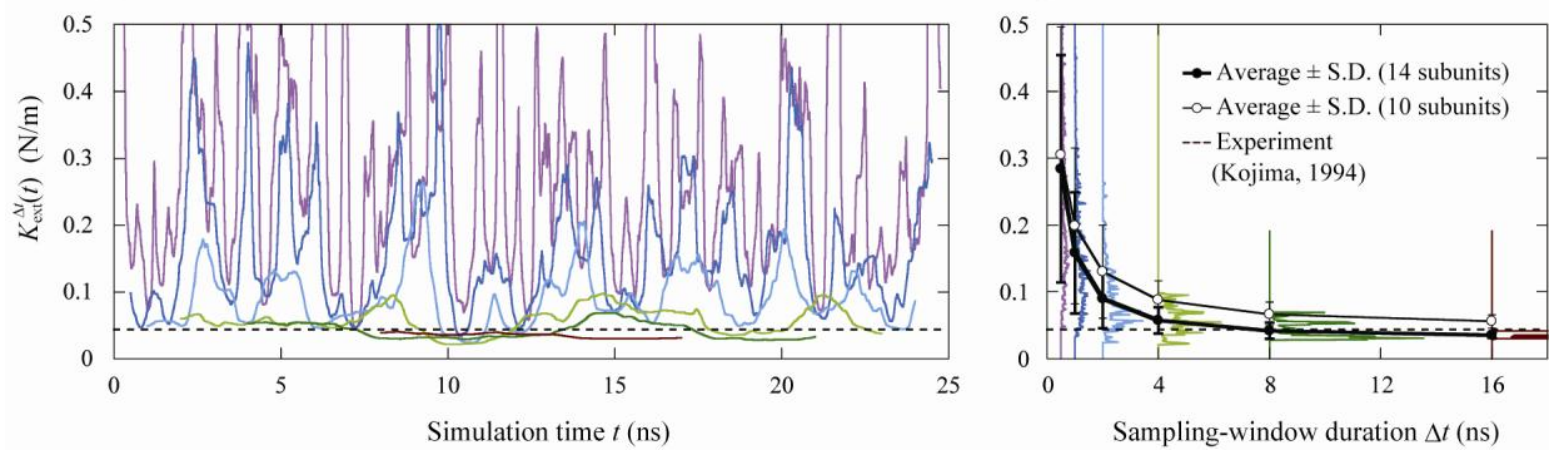

2

$3 \quad$ Figure 4: Variance of filament length and extensional stiffness during equilibrium determined for each sampling-window duration $\Delta t$. The experimentally determined stiffness (Kojima et al., 1994) is represented by the dashed lines. (A) shows the change in variances of

6 the filament length $\left\langle\Delta L^{2}(t)\right\rangle_{\Delta t}$. (B) shows the change in extensional stiffness $K_{\mathrm{ext}}^{\Delta t}(t) .(\mathrm{C})$

$7 \quad$ shows the distribution of extensional stiffness $K_{\mathrm{ext}}^{\Delta t}(t)$. 

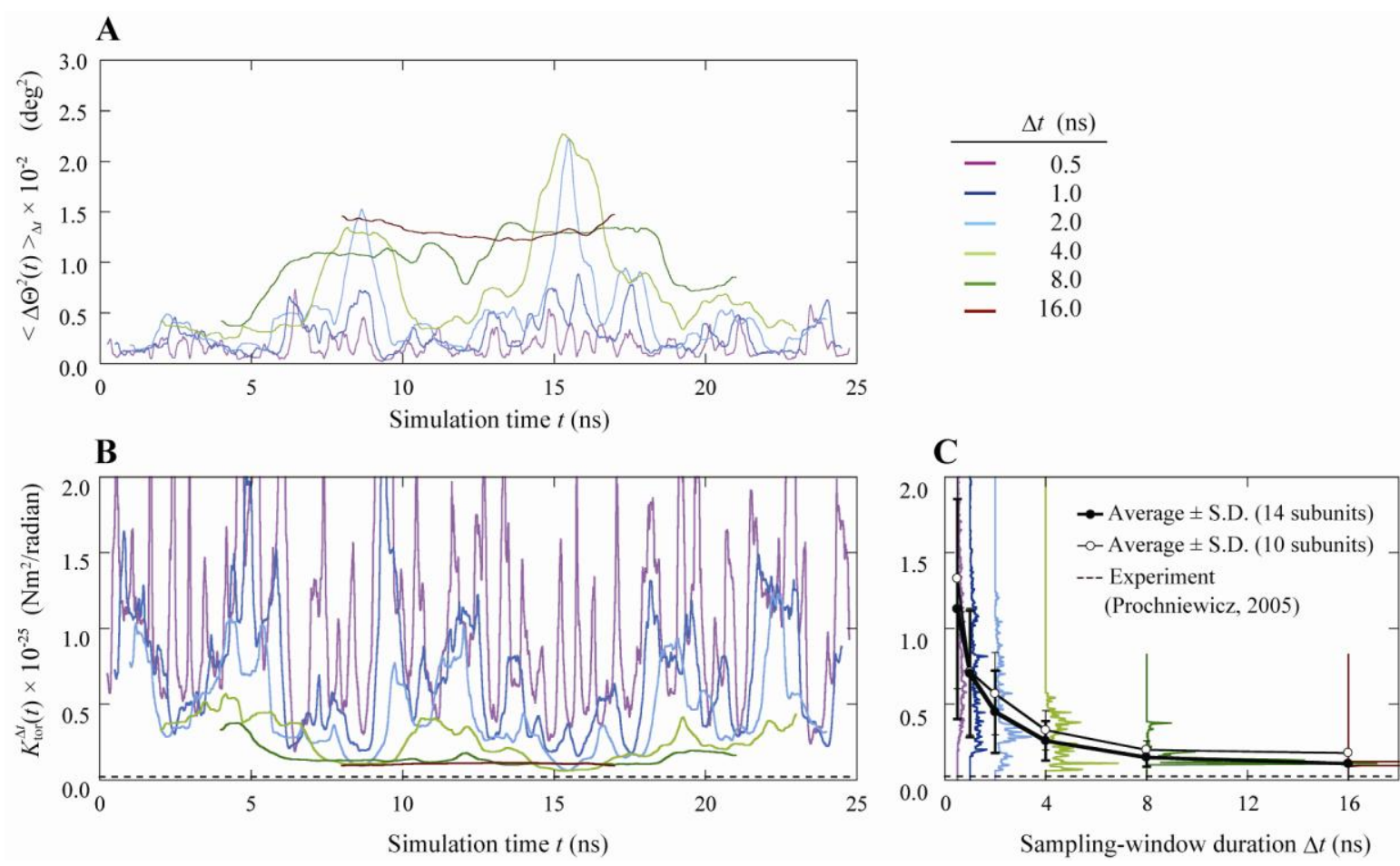

Figure 5: Variances of the twist angle and torsional stiffness during equilibrium determined

3 for each sampling-window duration $\Delta t$. The experimentally determined stiffness

4 (Prochniewicz et al., 2005) is represented by the dashed lines. (A) shows the change in the

5 variances of the twist angle $\left.<\Delta \Theta^{2}(t)\right\rangle_{\Delta t}$. (B) shows the change in torsional stiffness $K_{\text {tor }}^{\Delta t}(t)$.

6 (C) shows the distribution of torsional stiffness $K_{\text {tor }}^{\Delta t}(t)$. 